\title{
Alteración del Ecosistema en la Ciénega de las Quintas en Cartagena - Colombia ${ }^{54}$
}

\author{
Alteration of the Ecosystem in the Swamp of Las Quintas in Cartagena - Colombia \\ ${ }^{a}$ Édison Faciolince Gómez, Elfa Luz Mejía Mercado ${ }^{b}$ \\ a edfago17@hotmail.com “Semillero Políticas Públicas Participación y Desarrollo, Programa de Derecho, Universidad de Cartagena. Cartagena, \\ Colombia. \\ bedfago17@hotmail.com “Grupo de Investigación Conflicto y Sociedad, Programa de Derecho, Universidad de Cartagena. Cartagena, Colombia. \\ Forma de Citar: V.M. López “Teoría Jurídico - Económica del Daño Moral para la Indemnización de la Responsabilidad Estatal”, Rev. Saberes, \\ Vol. 13, No. 02, pp. $75-80,2020$.
}

Recibido: 24/03/2020 Evaluación: 28/05/2020 Aceptado: 30/06/2020 DOI: https://doi.org/10.25213/1794-4384/1302.0010

\section{Resumen}

El 22 de enero de 1978, hace 42 años, se inauguró el Mercado de Bazurto, ubicado en el centro de la ciudad de Cartagena entre la Avenida Pedro de Heredia y la Avenida del Lago. Esto significó un gran progreso para la ciudad puesto que se dio una mejor organización del Distrito. Sin embargo, la falta de controles ambientales por parte de las administraciones de la ciudad, ha permitido que durante ese mismo tiempo se creara un modelo mental entre los comerciantes, vendedores, pescadores y administradores del mercado, el cual se resume en que es culturalmente normal que este espacio destinado al comercio sea sucio y genere cantidades de basura diariamente que alteran el ecosistema.

Las altas tasas de contaminación, impactan de manera agresiva contra el ecosistema más próximo a la plaza de mercado, la Ciénega de las Quintas, la cual se conecta con todos los puntos fluviales de la Cartagena contaminando ampliamente la ciudad. La Ciénega está ubicada en la avenida del lago, es reconocida por ser el hogar del ecosistema marino costero conocido como manglar. La importancia de estos definida por el Ministerio de Medio Ambiente, radica en que protegen a una cantidad de organismos en sus troncos, raíces o en el fango y además que reduce el impacto de mareas y constituye una barrera natural de amortiguamiento que protege a las costas de marejadas y vientos huracanados a manera de cortina rompe vientos.

\section{Palabras Clave}

Manglar, medio ambiente, contaminación, nicho ecológico, ecosistemas.

\begin{abstract}
On January 22, 197842 years ago, it opened the Mercado of Bazurto, located in the center of the city of Cartagena between Avenue Pedro de Heredia and Avenue del Lago. This was a big step forward in the city, which is the main organizer of the district, nevertheless, the lack of environmental controls for the administrators of the city, and which will allow a mental model between merchants, vendors, fisherman and administrators of the market, which can be summed up in that it is culturally normal that this is a space intended for trade in sea and a generation of garbage daily that alter the ecosystem.

The high pollution rates aggressively impact the ecosystem closet to the market square, the Swamp of Las Quintas is located on the avenue of the lake, it is recognized as the home of coastal marine ecosystem known as mangrove. The importance of these defined by the Ministry of Environment, is
\end{abstract}

Autor para correspondencia: Correo electrónico edfago17@hotmail.com

(C) 2020 Fundación Universitaria Antonio de Arévalo - UNITECNAR. Este es un artículo bajo la licencia CC BY-NC-ND (http://creativecommons.org/licenses/by nc-nd/4.0/)

\footnotetext{
54 Ponencia presentada en el marco del Congreso de Investigación Científica en Ciencias Jurídicas y Económicas
} 
that they protect a number of organisms in plus $\operatorname{logs}$, roots or in the mud and also that it reduces the impact of tides and constitutes a natural buffer that protects the coast of swells and hurricane winds as curtain breaks winds.

\section{Keywords}

Mangrove, environment, pollution, ecological niche and ecosystems.

\section{Introducción}

Colombia ha registrado un marco normativo para velar por la protección de los cuerpos de agua y ecosistemas que habitan en ella, de esta forma a través de, el decreto 1715 de 1978 que según lo establecido por el Código Nacional de los Recursos Naturales Renovables y de Protección al Medio Ambiente (Decreto Ley 2811 de 1974), la comunidad tiene derecho a disfrutar de paisajes urbanos y rurales que contribuyan a su bienestar físico y espiritual. De igual forma en la Ley 99 de 1993, se establecieron los siguientes principios protectores del medio ambiente: la biodiversidad del pais por ser patrimonio Nacional y de interés de la humanidad deberá ser protegida prioritariamente y aprovechada en forma sostenible. La acción para la protección y recuperación ambientales del país, es una tarea conjunta y coordinada entre el Estado, la comunidad, las organizaciones no gubernamentales y el sector privado.

Existen otras normatividades como son la Ley 357 de 1997 por medio de la cual se aprueba la "Convención Relativa a los Humedales de Importancia Internacional especialmente como Hábitat de Aves Acuáticas"; la Ley 1259 de 2008 por medio de la cual se instaura en el territorio nacional la aplicación del comparendo ambiental a los infractores de las normas de aseo, limpieza y recolección de escombros y se dictan otras disposiciones; la Ley 1333 de 2009 por la cual se establece el procedimiento sancionatorio ambiental y se dictan otras disposiciones y por último la ley 1801 de 2016 donde se establece el Código de Policía.

Toda esta reglamentación instaurada en el estado colombiano permite la protección de los recursos naturales y el medio ambiente. En esta ponencia analizaremos la implementación de esta normatividad en la Ciudad de Cartagena, una de las ciudades con más riqueza de especies y recursos hidrográficos, debido a que, se encuentra rodeada por cuerpos de agua, que se interconectan de manera interna y externa, estos cuerpos de agua le han dado históricamente reconocimiento como una de las ciudades más bellas de Colombia y esto le ha permitido ser un centro turístico atractivo que genera muchos ingresos al año.

Los cuerpos de agua mencionados anteriormente son el hogar del ecosistema marino costero conocido como "manglar", el mangle "es un arbusto de las rizofóreas, de tres a cuatro metros de altura, cuyas ramas largas y extendidas dan unos vástagos que descienden hasta tocar el suelo $y$ arraigar en él. Tiene hojas pecioladas, opuestas, enteras, elipticas, obtusas y gruesas; flores axilares de cuatro pétalos amarillentos; fruto seco de corteza coriácea, pequeño y casi redondo y muchas raices externas en parte”. (Hogarth, 1999).

Por otra parte, los mangles tienen una clasificación, dependiendo de su ubicación, especies, y acceso al agua. De esta forma tenemos en Cartagena, los mangles de la Ciénega de la Virgen, clasificados según el EPA como mangle colorados (Hervario Rizophora Mangle), mangle negro (Avicennia germinans) y mangle bobo (Laguncularia racemosa) en el caño de Juan Angola, mangles Zaragoza en la laguna de Chambacu y el mangle rojo en la Ciénega de las Quintas (Hervario Rizo hora Mangle).

"La Ciénaga de la Quinta hace parte del sistema de caños y lagos internos de la ciudad de Cartagena y se encuentra localizada entre el puente Jiménez y el puente de Bazurto y se caracteriza por tener anchos entre 288 y $477 \mathrm{~m}$, con profundidad de $2,25 m$ y un área total del espejo de agua de 30 hectáreas. La ciénaga, se localiza en la latitud $10^{\circ} 24^{\prime} 46^{\prime \prime} N$ y longitud 75³1 '46”"' E Tirado, O.; Manjarrez, G.; Díaz. 
Según los estudios realizados por CARDIQUE ${ }^{55}$ se ha establecido que el mangle rojo ubicado en la Ciénega de las Quintas se caracteriza porque pueden alcanzar hasta 35 metros de altura, sus hojas tienen de 8 a $10 \mathrm{~cm}$ de longitud y $4^{\mathrm{a}} 5 \mathrm{~cm}$ de ancho. "Las funciones de este ecosistema es que son salas cunas de alevinos y otras especies, que hacen de esta ciénaga un ecosistema rico en especies de flora y de fauna. Se resalta que esta flora tiene una función depuradora de las aguas altamente contaminadas de la ciénaga. (EPA, Cartagena, 2006);

Sin embargo, toda esta maravilla del ecosistema, se ha venido viendo afectada, por la constante tasa de contaminación emitida por el Mercado de Bazurto, el cual solo en enero del año 2018 según el informe realizado por el periódico El Espectador, emitió más de 1.000 toneladas de basura, sin que exista un control de los vertimientos. Además, la Ciénaga de la Quinta es utilizada como la principal zona de disposición de residuos de los comercializadores de pescado, de carne y de pollo, quienes indiscriminadamente vierten en esta las vísceras y demás productos, contaminando el agua y generando olores ofensivos. El volumen de residuos producido por el Mercado de Bazurto alcanza cerca de $60 \mathrm{~m} 3$ o 15 toneladas (Disposición de basuras en Cartagena, 2007).

Por tal motivo, los habitantes del barrio aledaño, Martínez Martelo, instauraron una Acción Popular en el año 2010, en la cual el Tribunal Administrativo de Bolívar aceptó y ordenó a la Alcaldía de Cartagena, proceder con los estudios y reubicación de la plaza, sin embargo, por la interinidad que ha padecido la ciudad no ha podido hacer efectivo el cumplimiento del fallo judicial.

Ante la ausencia de autoridad, los comerciantes, pescadores y vendedores se han acostumbrado a que Bazurto sea un lugar en el cual se pueden violar todas la condiciones ambientales, tanto así, que la falta de salubridad y contaminación emitida por estos actores, impacta contra el nicho ecológico del sector, de tal manera que nace el interrogante ¿La alteración en la cadena alimenticia por el suministro de desechos a las

\footnotetext{
55 Corporación Regional que funge como autoridad Ambiental (Corporación Regional del Canal del Dique).
}

especies claves del ecosistema de la Ciénega de las Quintas afecta de manera directa el consumo humano y la subsistencia del ecosistema?

\section{Metodología}

La metodología utilizada es de carácter cualitativa jurídica desde la perspectiva de la eficacia de la normatividad en la protección del medio ambiente, con base a la recolección e interpretación comparativa de datos, a través de la observación directa de los documento obtenidos en la caracterización ambiental de la Ciénaga de la Quinta localizada en Cartagena de Indias, Colombia, año 2009 - 2010 elaborada por Omar Tirado, Ganivet Manjarrez, Claudia Díaz y el informe sobre la caracterización biofísica y socioeconómica rápida de las condiciones ambientales de la ciénaga las quintas, Cartagena, Bolívar elaborado por el Establecimiento Público Ambiental de Cartagena EPA Cartagena en el año 2016, con la evaluación de los datos en ambos estudios se establecerá si existe daño ambiental que en largo plazo pueda afectar a los ciudadanos consumidores de los productos del mercado de Bazurto.

\section{Resultados y Discusión}

Los resultados encontrados en los estudios resaltados anteriormente arrojaron que el sector de la Ciénega de Las Quintas, se encuentra conformado por grupos pesqueros y una diversa flora y fauna. Se determinó que en la zona actúan por lo menos tres (3) grupos o asociaciones de pescadores: La Asociación de Pescadores del Barrio Chino, con 18 miembros activos; la Asociación de Pescadores de Bazurto con 16 miembros activos y la Asociación de pescadores de Ceballos con 22 miembros activos. Sin embargo, los pescadores identificados por faenar en el área en referencia con atarraya no registraron estar asociados en ninguna organización. ${ }^{56}$

Dentro de las especies principalmente capturadas con este arte de pesca, destacan: lisa (Mugil Incilis), anchova (Mugil Curema), lebranche (Mugil Liza), mojarra huesuda (Diapterus

\footnotetext{
${ }^{56}$ Informe final sobre la caracterización biofísica y socio- económica de las condiciones ambientales de la ciénaga Las - Quintas, Cartagena, Bolívar. EPA Concepto técnico CPT-CAM-007-16
}

Página | 77 
Rhombeus), mojarra rayada (Eugerres Plumier) y róbalo (Centropomus Undecimalis). Estas especies generalmente son de tallas pequeñas.

En el informe se registraron las siguientes especies:

- A partir de las muestras colectadas, en la ciénaga Las Quintas, se identificaron un total de 73 morfoespecies, 33 géneros, 27 familias, 20 órdenes, seis clases y cinco phylum: Bacillariophyta, Miozoa, Cyanobacteria, Euglenophyta = Phylum Euglenozoa, Chlorophyta. Dos morfo-especies, solo se lograron clasificar taxonómicamente hasta el nivel de phylum: Bacillariophyta y Chlorophyta; y tres se clasificaron hasta el nivel de clase: Bacillariophyceae, Dinophyceae y Cyanophyceae.

- Se encontró un total de nueve phylum (Annelida, Arthropoda, Chaetognatha, Chordata, Ciliophora, Cnidaria, Foraminifera, Nematoda y Rotifera), de los cuales 15 fueron llevadas a nivel de especie, seis a género, ocho a nivel de familia y el restante perteneció a estadios larvales, juveniles o fragmentos (Anexo 2), para un total de 41 taxa. Se registraron ocho taxa meroplanctónicos (dos larvas de poliquetos, una hidromedusa, dos larvas de cirripedios, un nematodo, un juvenil y un huevo de pez) y 33 taxa holoplanctónicos.

- En los 14 puntos de muestreo se realizaron 19 lances de atarraya, colectándose un total de 15 ejemplares de peces, un crustáceo y un molusco.

- Los 15 ejemplares capturados e identificados pertenecen a tres especies de peces marinos: Robalo (Centropomus undecimalis), Mojarra rayada (Eugerres plumier) y Mojarra huesuda (Diapterus rhombeus).

Dentro de los lances efectuados se recolectó en el P06 de muestreo un molusco, Borcellar leachii o liebre de mar (ver ficha taxonómica en el Anexo 4), el cual es un opistobranquio bentónico de tamaño medio que se ubica dentro de la orden Anaspide, familia Aplysiidae.

- Se colectó un organismo de la especie Callinectes sapidus, familia Portunidae, orden Decapoda comúnmente conocida como jaiba. Estos organismos omnívoros pueden encontrarse en aguas dulces, estuarinas y marinas, preferiblemente en lugares fangosos y arenosos (Escobar y Giraldo. 1993).
- Aves: se observaron varias especies de aves perchadas en las ramas y copa de los mangles, entre las cuales se destacan por su abundancia en el ecosistema, la garza blanca (Ardea alba), la cual fue vista en bandadas y algunos individuos solitarios; pelícano (Pelecanus occídentalis); y maría mulata (Quíscalus mexicanus), especies que mayormente se alimentan de los residuos orgánicos de la venta de pescados, y que encuentran refugio en el manglar.

Analizando la fauna del ecosistema del sector, podemos inferir que la especie clave dentro de este nicho es el robalo, se ha demostrado que el róbalo se alimenta de manera oportunista, cambiando de presas en relación con el tipo de hábitat que ocupan (Blewett et al., 2006).

Se entiende especie clave como aquella especie que ejerce una influencia directa sobre los otros miembros de la comunidad sin proporción a su abundancia o densidad. Al remover una especie clave la comunidad cambia drásticamente y el ecosistema sucumbe ante la presión del disturbio (Paine 1966). "La presencia del robalo es destacada en ciénagas, en donde encuentra un hábitat adecuado para su desarrollo y crecimiento, por lo cual, su captura y frecuencias son mayores en estos lugares". (Morales, 1975).

El estado de conservación actual del robalo, lo posiciona como una especie vulnerable de acuerdo con la Resolución 192 de 2014 del MADS, sobre especies silvestres amenazadas de la diversidad biológica colombiana, así como el Libro Rojo de peces de Colombia (Mejía y Acero, 2002).

Sin embargo, con la caracterización realizada en el año 2016 se obtuvo ya existe evidencia de las consecuencias de la degradación del hábitat sobre los róbalos. Se ha informado que la diversidad de la dieta en estuarios menos degradados es mayor que estuarios más degradados y la importancia de las presas más relevantes es desproporcionadamente más alta en los estuarios más degradados (Adams et al., 2009).

El problema que causa la degradación del hábitat de las especies anteriormente descritas es la alta tasa de contaminación emitida por el Mercado de Bazurto, la caracterización del año 2010 y 2016 consideran que uno de los principales factores de degradación de la ciénaga, es la mala disposición de sus residuos líquidos y sólidos, lo cual ha

Página | 78 
causado deterioro de la calidad del agua y los sedimentos, afectación a la biota asociada, y deterioro estético de la ciénaga Las Quintas. Residuos sólidos como plásticos, icopor, restos de material orgánico son arrojados al cuerpo de agua directamente o transportados por los vientos, acumulándose de forma evidente en la ribera de la ciénaga, (Cartagena EPA Cartagena en el año 2016). Esta problemática resaltada propende en que se produzcan varios fenómenos que afecten tanto al ecosistema como a la población aledaña al sector de la plaza de mercado, tales efectos pueden ser el aumento de los agentes infecciosos y enfermedades. Que causan importantes de morbilidad y mortalidad en animales libres y en cautiverio. Algunos agentes infecciosos de los animales silvestres son únicos para ciertas especies o grupos, pero también pueden tener potencial patogénico hacia otros animales y personas. (Fowler, 1986). A su vez, tales enfermedades pueden afectar a las personas, que están en constante cercanía con estas especies, poniendo en riesgo su vida y la de sus familiares.

La discusión de los datos observados en la comparación radica en cuáles deberían ser las medidas a tomar por la administración de Cartagena para frenar este deterioro ambiental que se presenta en el sector de la Ciénega de las Quinta, a pesar de los ciudadanos haber agotados recursos jurídicos, como la Acción Popular, inclusive con la existencia del fallo judicial a favor de la reubicación del mercado, no se han visto cambios hasta el año 2020, donde es constante el arrojo de desperdicios tales como viseras de cerdo, pollo y res como residuos sólidos y líquidos hacia la Ciénega que son en muchas ocasiones consumidos por la fauna, disminuyendo su ciclo de vida y así cada vez más ir deteriorando el ecosistema en el que habitan y comparten con los seres humanos actores activos de la contaminación.

\section{Conclusiones}

Las reflexiones obtenidas del análisis de los datos permite afirmar al autor que las altas tasas de contaminación ambientales registradas en el Mercado de Bazurto son un problema que afecta tanto a los barrios aledaños como a el ecosistema más cercano a este sector, estos problemas se pueden resumir en que el constante vertimiento de desechos dentro de la Ciénega de las Quintas está produciendo contaminación y alteración del $\mathrm{PH}$ del agua, reduciendo las especies dentro del ecosistema de mangle rojo. Esta reducción y remoción de especies claves está destruyendo la cadena alimenticia alterando el ecosistema. Produciendo que muchas especies dejan de un lado realizar las actividades de caza, para consumir los residuos arrojados por las personas que desarrollan sus actividades diarias en el Mercado.

Por otra parte, la alteración del ecosistema, puede causar cambio en la fauna que encontramos en el humedal, disminuyendo la actividad económica de los pescadores, reflejada en la disminución de los ingresos obtenidos de la pesca obligándolos o a cambiar de oficio o a buscar un nuevo lugar donde explotar sus actividades.

Por último, esta alteración puede llegar a producir enfermedades a los habitantes del sector y a los ciudadanos que desarrollan sus actividades en la plaza de mercado, puesto que la constante exposición a estos residuos y animales enfermos por la contaminación pueden llegar a desarrollar enfermedades que deterioren su salud rápidamente.

En conclusión son urgentes que se tomen medidas por la administración de la ciudad de Cartagena, para que se sancione a cualquier persona que contamine el sector, conforme a lo desarrollado en la Ley 1259 de 2008, por medio de la cual se instaura en el territorio nacional la aplicación del comparendo ambiental a los infractores de las normas de aseo, limpieza y recolección de escombros; y se dictan otras disposiciones; y por último la Ley 1333 de 2009 Por la cual se establece el procedimiento sancionatorio ambiental y se dictan otras disposiciones. Además de que se exija el cumplimiento por parte de Los concejos municipales y distritales quienes deberán destinar anualmente a las corporaciones autónomas regionales o de desarrollo sostenible del territorio de su competencia, para la protección del medio ambiente y los recursos naturales renovables, el porcentaje ambiental del impuesto predial, sobretasa de medio ambiente, de que trata el artículo 44 de la Ley 99 de 1993.

\section{Referencias Bibliográficas}

Adams, A.J., R.K. Wolfe y C.A. Layman. 2009. Preliminary examination of how human-driven freshwater flow alteration affects trophic ecology Página | 79 
of juvenile snook (Centropomus undecimalis) in estuarine creeks. Estuar. Coast. 32: 819-828.

Blewett, D.A., R.A. Hensley y P.W. Stevens. 2006. Feeding habits of common snook, Centropomus undecimalis, in Charlotte Harbor, Florida. Gulf Carib. Res. 18: 1-13

Beltrán Reales y Suárez Esquivia:(2010) Diagnóstico ambiental de los cuerpos internos de agua de la ciudad de Cartagena de Indias, Edición electrónica gratuita.

El decreto 1715 de 1978 que según lo establecido por el Código Nacional de los Recursos Naturales Renovables y de Protección al Medio Ambiente (Decreto Ley 2811 de 1974).

EPA, 2016. Informe final sobre la caracterización biofísica y socio- económica de las condiciones ambientales de la ciénaga Las - Quintas, Cartagena, Bolívar. EPA Concepto técnico CPTCAM-007-

16/observatorio.epacartagena.gov.co/ftpuploads/ga-eco-caracterizacion-biofisicasocioeconomica-cienaga-de-las-quintas.pdf Establecimiento público ambiental - EPA Cartagena, ecosistemas manglar de la Ciénega de la Virgen Juan Polo o de Tesca. http://observatorio.epacartagena.gov.co/gestionambiental/ecosistemas/proyecto-cienaga-de-lavirgen/cienaga-de-la-virgen/

El Espectador, revisado 2020, Bazurto el corazón de la contaminación en Cartagena// www.elespectador.com/noticias/nacional/bolivar/ mercado-de-bazurto-el-corazon-de-lacontaminacion-en-cartagena-articulo-838241

El Universal, 2019, Cristian Pájaro www.eluniversal.com.co/suplementos/facetas/lavida-antes-del-mercado-de-bazurto-JF733964

Hogarth p. J. 1999. The biology of mangroves. Oxford Univ. Press; Oxford, UK. Ix +228 p. ISBN 0-19-850222-2. Paperback.

Morales, J. 1975. Estudio biológico-pesquero del robalo (Centropomus undecimalis Bloch, 1792) en la ciénaga Grande de Santa Marta. Tesis para optar por el título de Biólogo Marino. Univer. De Bogotá Jorge Tadeo Lozano. Bogotá, Colombia. 81p.
Mejía, L.S. y A. Acero. (Eds.). 2002. Libro rojo de peces marinos de Colombia.

INVEMAR, Instituto de Ciencias NaturalesUniversidad Nacional de Colombia, Ministerio de Medio Ambiente. La serie Libros rojo de especies amenazadas de Colombia. Bogotá, Colombia.

Ministerio del Medio Ambiente, revisado 2020. www.minambiente.gov.co/index.php/component/ content/article?id=412:plantilla-bosquesbiodiversidad-y-servicios-ecosistematicos-14

La Ley 99 de 1993: Se establecieron los siguientes principios protectores del medio ambiente: La biodiversidad del país por ser patrimonio Nacional y de interés de la humanidad deberá ser protegida prioritariamente y aprovechada en forma sostenible.

La Ley 357 de 1997 por medio de la cual se aprueba la \&quot;Convención Relativa a los Humedales de Importancia Internacional especialmente como Hábitat de Aves Acuáticas\&quot;.

La Ley 1259 de 2008 por medio de la cual se instaura en el territorio nacional la aplicación del comparendo ambiental a los infractores de las normas de aseo, limpieza y recolección de escombros; y se dictan otras disposiciones.

La Ley 1333 de 2009 por la cual se establece el procedimiento sancionatorio ambiental y se dictan otras disposiciones.

La ley 1801 de 2016 donde se establece el Código de Policía.

Robert T. Paine Food Web Complexity and Species Diversity, the American Naturalist, Vol. 100, No. 910 (Jan. - Feb., 1966), pp. 65-75

Zonificación de manglares en la jurisdicción de CARDIQUE, 2007.2 MI-0275. https://cardique.gov.co/ 